\title{
Application of Circuit Modeling in the Study of Spark Formation During Electroerosion Treatment
}

\author{
Artur Porvatov ${ }^{1,4}$, Mikhail Kozochkin ${ }^{1}$, Ilya Minin ${ }^{1,2,3,4}$, Andrey Maslov ${ }^{1}$, Vladimir Kuptsov ${ }^{3}$, Marian Morozkin ${ }^{3}$, Vladimir \\ Kuznetsov $^{5}$, Marko Leonesio ${ }^{6}$, Alexander Zelensky ${ }^{1}$, Petr Pivkin ${ }^{1,2,3,4}$ \\ ${ }^{1}$ Dept. of High-Efficiency Processing Technologies, Moscow State University of Technology “STANKIN”, RU-127055, Moscow, \\ Russia, \\ ${ }^{2}$ Dept. of Cutting Tools and Shaping Technologies, Moscow State University of Technology “STANKIN”, RU-127055, Moscow, \\ Russia; \\ ${ }^{3}$ Lab. of Micromachining Technologies, Moscow State University of Technology “STANKIN”, RU-127055, Moscow; Russia; \\ ${ }^{4}$ Center of Cognitive Technologies and Machine Vision, Moscow State University of Technology “STANKIN”, RU-127055, Moscow; \\ Russia; \\ ${ }^{5}$ Moscow Polytechnic University, RU-107996; Moscow; Russia; \\ ${ }^{6}$ Institute of Industrial Technologies and Automation, Italian National Research Council, IT-00187 Rome, Italy.
}

\begin{abstract}
Electrical discharge machining (EDM) of a workpiece is a complex, fast-flowing process characterized by alternating (intermittent) states: short circuit, idle and spark formation. The discontinuity of the EDM process means that the processing is carried out in single pulses, which are formed by a special pulse generator. The parameters of the generator pulses can be divided into time and electrical. The time period and duration of the pulses, as well as the difference between these two parameters (the "silence" interval), are considered temporary. The electric ones include the amplitude value of the voltage, the maximum permissible current, and the polarity of the pulses. in addition, depending on the device of the generator, the pulses can be composite, for example, have an igniting pulse with a higher voltage and a lower current than the main (working) pulse. In this work, we have developed a interelectrode gap model that allows not only to obtain values of electrical parameters, but also to evaluate and to optimize the electrical parameters of materials being processed with known electrical properties. The key advantage of this model is its modularity, which allows to add new functional blocks, which describe external and internal influences, for example, the concentration of erosion products, uneven electrical conductivity of the workpiece, and others, without changing its structure.
\end{abstract}

\section{Introduction}

The EDM process is influenced by various factors such as the electrical conductivity of the workpiece and liquid, the state of the electrode, the parameters of the generator and others. One of them is the state of the interelectrode gap (IEG). The state of the IEG has concentrated most of the parameters that are difficult or impossible to control in the EDM process, so its assessment is carried out indirectly, for example, by current. However, this does not give a complete picture of what is happening with EDM, and even more so does not allow you to estimate in advance certain EDM parameters necessary for setting up the generator [1].

The IEG is the distance between the electrode and the workpiece [2]. With EDM, the field strength in the IEG increases up to the discharge with a decrease in this distance. conditionally, depending on the interelectrode distance, the IEG can be in three states (fig. 1):

1) idling (XX), at $\mathrm{h} 2<\mathrm{IEG}<\infty$;

2) operating pulses (ri), at $h 1<$ ieg $<\mathrm{h} 2$;

3) short circuit (short circuit), at $0<$ ieg $<$ h1, where $\mathrm{h} 1$ and $\mathrm{h} 2$ are the lower and upper bounds for the occurrence of discharges, respectively.

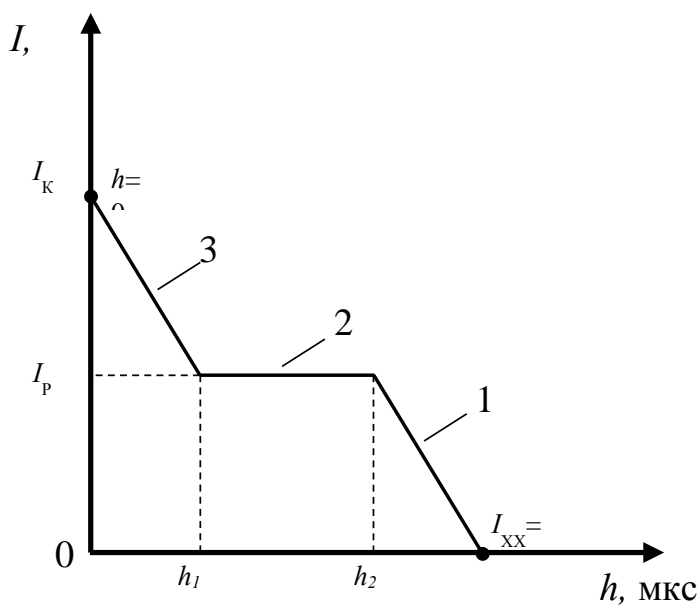

Fig. 1. Dependence of current (I) on the value of IEG (h) for EEP 
The IEG model should take into account the transition from one state, since the EEP process itself depends on it. The description of the IEG model can be simplified by introducing assumptions:

1) when $x x$ discharges are absent, there is no material removal and the IEG behaves like a dielectric;

2) when short-circuit discharges are present, but the removal of material does not occur and the IEG behaves as an ideal conductor.

Thus, the main interest is in area 2 (see Fig. 1), in which the material is removed.

In this area, a discharge occurs in the IEG (during the operation of the generator and the formation of current pulses), which takes place in 6 stages:

1) the appearance of an electromagnetic field between the electrode and the workpiece;

2) ionization of the fluid in the IEG;

3) formation of a conduction channel;

4) spark discharge-conversion of electrical energy into thermal energy (formation of a plasma channel) destruction of the material;

5) crater formation;

6) repeat steps 1-5.

At stage 4, a plasma channel is formed in the IEG and the material of the electrode and part is destroyed, which occurs unevenly for both of them and depends on the polarity of the applied voltage. Thus, it can be concluded that with an increase in voltage or a decrease in distance, the resistance of the IEG before discharge decreases sharply and increases after it. The increase in resistance is due to the ionization of the liquid in the IEG after discharge. the time associated with" de-ionization " (restoration of the IEG resistance after discharge), along with the pulse time, is often entered as a parameter for controlling the eep process, and the pulse repetition period is calculated as their sum. also, after the discharge, erosion products are formed, which reduce the resistance of the IEG and interfere with the passage of pulses between the electrode and the part. These two parameters can be combined in one concept - electrical strength.

\section{Results and Discussion}

The electrical strength of the working fluid (RJ) strongly depends on the presence of various impurities, including erosion products. Taking as a basis the concept of concentration, which describes the percentage of erosion products in the IEG from 0 to $100 \%$, it is possible to express the breakdown strength of RYE contaminated with erosion products as a function of the distance and concentration of erosion products between the electrode and the part [3-4]:

$$
\left\{\begin{array}{l}
h_{\text {МЭП }} \approx h_{n p}=\frac{U_{И}}{E_{n p}} \\
E_{n p .3 .}=E_{n p}\left(1-\frac{K_{3}}{100 \%}\right)
\end{array}\right.
$$

where the voltage pulse (In), EPR - electric strength of uncontaminated working fluid $(\mathrm{V} / \mathrm{m})$, map - value MEHP (m), p - value breakdown of IEG (m), KZ is the concentration of erosion products in the map (from 0 to 100\%), EPR.z. - tension breakout RJ contaminated with products of erosion.

Sometimes researchers use the schematic representation model of the IEG, shown in Fig. 2. Based on the current-voltage characteristic of the IEG [2], the circuit model of the IEG will be characterized by equivalent parameters: inductance Leq, resistance Req and capacitance Ceq (Fig. 2)

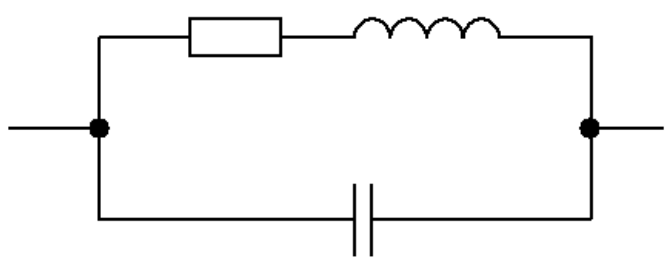

Fig. 2. Schematic model of IEG with equivalent $L_{\mathrm{eq}}, R_{\mathrm{eq}}, C_{\mathrm{eq}}$

Equivalent parameters $\left(L_{\text {eq }}, R_{\text {eq }}, C_{\text {eq }}\right)$ can be determined using the equations:

$$
R_{e q}=\frac{d}{\sigma \cdot S}
$$

and

$$
C_{e q}=\varepsilon_{0} \varepsilon_{r} \frac{S}{d}
$$

Where $S=\pi \cdot r_{\ni}^{2}$ is the area of the end of the cylindrical electrode ( $r \ni$ is the radius of the electrode), $d$ is the distance of the IEG, $\sigma$ is the specific conductivity of the medium, $\varepsilon \mathrm{r}$ is the permittivity of the medium, $\varepsilon \mathrm{r}=$ $8.854 \cdot 10-12 \mathrm{~F} / \mathrm{m}$ is the electric constant,

Knowing the processing parameters (the rate of change of current $d i / d t$ and the value of voltage $\left(\mathrm{V}_{\mathrm{L}}(\mathrm{t})\right)$ in the IEG zone), the equivalent inductance can be estimated by the formula

$$
V_{L}(t)=L(t) \frac{d t}{d t}
$$

However, this model does not take into account the fact that these parameters can change, since the geometry and mutual location of the workpiece and the electrode, as well as the concentration of erosion products in the IEG during the EEP process, change. It is also necessary to take into account the properties of the plasma channel formed during the passage of the discharge and the processing parameters set by the generator - pulse time, duty cycle, maximum and average current. In [3], the inductance of the plasma channel that occurs when the current is discharged as estimated as 


$$
L(t)=2 d\left(\ln \left(\frac{2 d}{a(t)}\right)-0.75\right)
$$

where $L(t)$ is the inductance of the plasma (ngn), $d$ is the IEG, and $a(\mathrm{t})$ is the radius of the plasma channel ( $\mathrm{mcm})$, depending on the current and properties.

$$
a^{2}(t)=\left(\frac{4}{\pi^{2} \rho_{0} \zeta \sigma}\right)^{1 / 3} \int_{0}^{t} i^{2 / 3}(t) d t
$$

where $i$ is the channel current (A), $\mathrm{p}_{0}$ is the initial gas density, $\sigma$ is the conductivity, and $\zeta$ is the temperature coefficient [4].

As can be seen from (4), the current values of current and voltage, which are largely determined by the EEP pulse generator, also affect the equivalent electrical parameters of the MEP. Thus, the MEP model should additionally include a model of a pulse generator, the main parameters of which are:

- Operating current I, A;

- Operating voltage $\mathrm{V}, \mathrm{V}$;

- operating frequency $\mathrm{f}, \mathrm{Hz}$ :

$$
f=\frac{1}{T}=\frac{1}{t_{\text {on }}+t_{\text {off }}}
$$

where $\mathrm{T}$ is the period equal to the sum of the pulse durations $\left(\mathrm{t}_{\mathrm{on}}\right)$ and its absence $\left(\mathrm{t}_{\mathrm{off}}\right)$;

- $\quad$ pulse duty cycle, $q$, or the inverse of it, $D_{u}$ (duty cycle):

$$
D_{U}=\frac{1}{q}=\frac{t_{o n}}{t_{o n}+t_{o f f}}
$$

- $\quad$ pulse fill factor for a discharge of duration ti, which may not coincide with ton:

$$
D_{I}=\frac{t_{I}}{t_{o n}+t_{o f f}}
$$

Modern generators are complex power electronics devices with a large number of feedbacks to analog and digital controllers [5]. However, the principle of operation of most of them is similar and consists in the formation of an output pulse with the specified parameters (see above). In this regard, the generator consists of:

1) voltage source;

2) of control unit;

3) one or more managed keys.

Construction of the model and simulation was carried out in the environment of circuit modeling LTspice XVII (Linear Technology). Figure 3 shows a block diagram of the model for studying the EEE process, as well as a schematic model of the MEP (Block 4) based on formulas (1), (2) and (3).

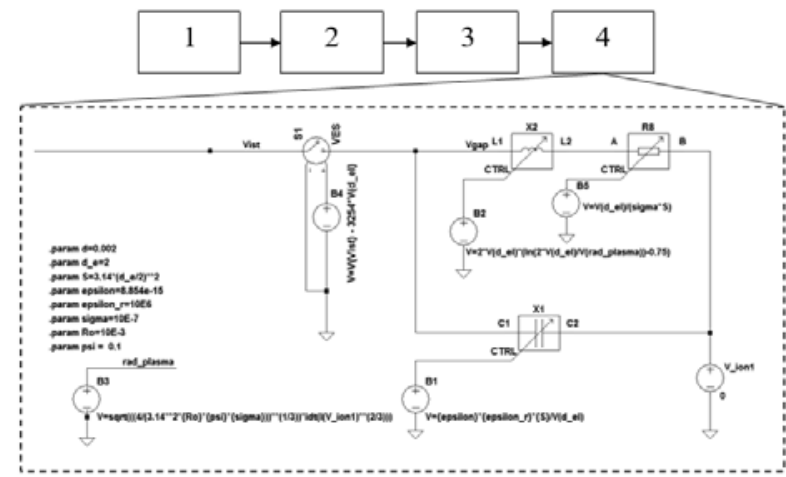

Fig. 3. The block diagram model MEP generator: voltage source (1), the control unit (2), two managed key (3), schematic model of interelectrode gap (4).

Model of inductance, capacitance and resistance in block 4 - MEP (Fig. 3) described in the form of macromodels using the Spice language, the parameters of which vary according to the given laws (see formulas above) using a controlled voltage source.

The generator circuit is based on a half-bridge circuit

As a result of modeling, the time dependences of current and voltage were obtained, as well as the interdependence of the conductivity of the MEP and the current at breakdown, the values of the MEP and the breakdown voltage, and many others. Figure 4 shows an example of the time dependence of current (I (R2)) and voltage ( $\mathrm{V}$ (vist)) with a linear change in the MEP value from 1000 microns (XX from 0 to $2.5 \mathrm{~ms}$ ) to 0 (shortcircuit for $7 \mathrm{~ms}$ ) and vice versa.

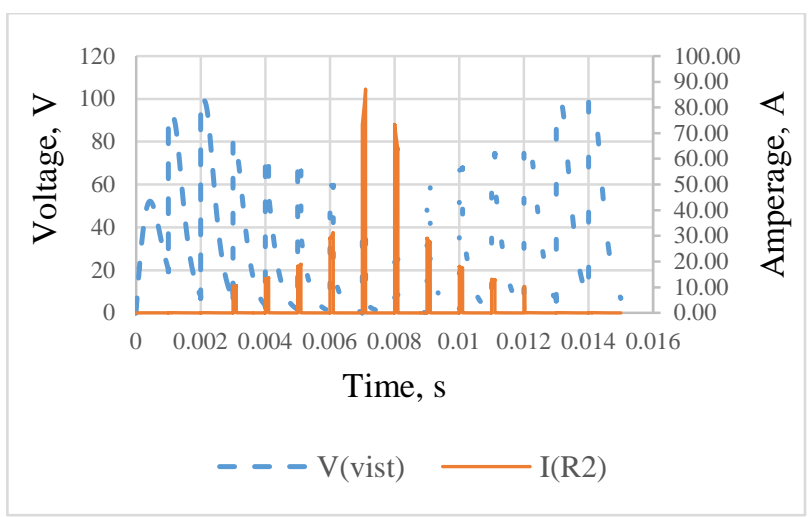

Fig. 4. Simulation results for linear reduction and increase of the interelectrode gap (voltage-dashed line, current - solid).

The data obtained in the simulation are in good agreement with other experimental data [6]. Taking into account the peculiarities of the behavior of current and voltage during EEE, it is possible to evaluate the effectiveness of a particular mode of processing various materials.

The adequacy of the obtained results of circuit modeling was evaluated by comparison with the results of experimental studies on the developed experimental stand [7-34]. Figure 5 shows a joint graph of the results of the experiment (a) and simulation (b) at the operating pulse. 


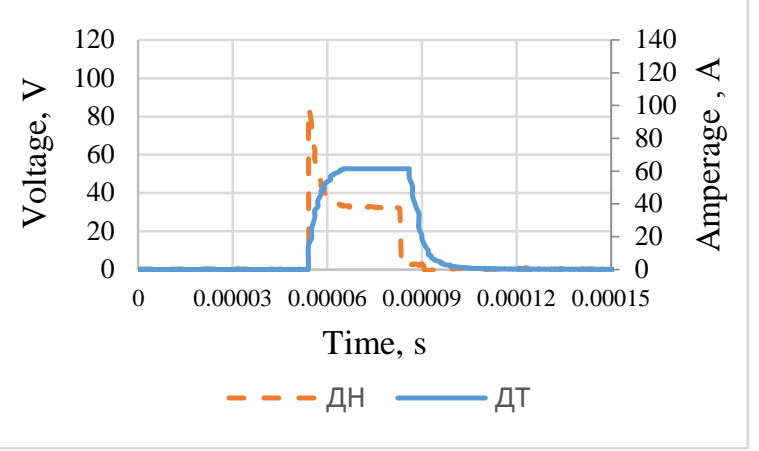

a)

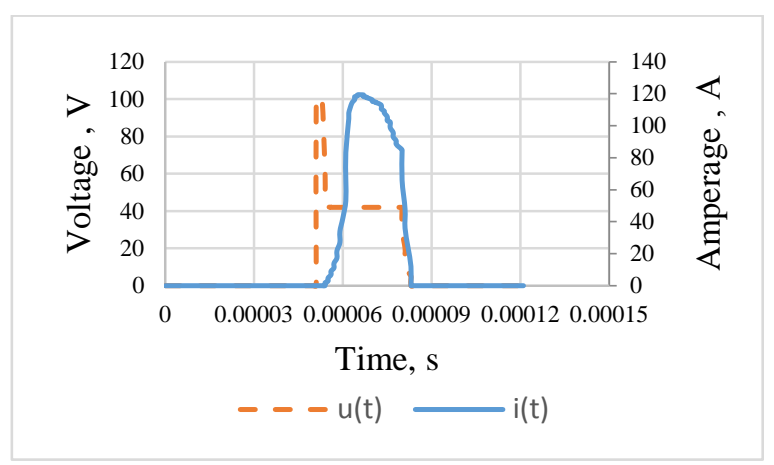

b)

Fig. 5. Comparison of the results of the experiment (a) and simulation (b) on the example of a single pulse at a working pulse.

As can be seen from Fig. 5, the voltage at RI (discontinuous graph) is the same in shape and mean values during simulation and experiment. small deviations are due to the characteristics of the generator of the experimental installation and parasitic inductances of the wires of this generator. The average values of the currents (solid graph) do not match. in this case, there is a limitation of the input range of the measuring system, in particular the analog-to-digital converter, so the current has a significant cut of the pulse tip.

\section{Conclusion}

The developed model allows not only to obtain values of electrical parameters, but also to evaluate and to optimize the electrical parameters of materials being processed with known electrical properties. The key advantage of this model is its modularity, which allows to add new functional blocks, which describe external and internal influences, for example, the concentration of erosion products, uneven electrical conductivity of the workpiece, and others, without changing its structure.

\section{Acknowledgments}

The study was carried out with the financial support of the Russian Foundation for Basic Research within the framework of scientific project No. 21-19-00790. This work was carried out using equipment provided by the Center of Collective Use of MSUT "STANKIN".

\section{References}

1. T.R. Raul, A. Saha, H. Majumder, et al., J Braz. Soc. Mech. Sci. Eng., 41, 300 (2019).

2. S.N. Grigoriev, M.A. Volosova, A.A. Okunkova, S.V. Fedorov, K. Hamdy, P.A. Podrabinnik, P.M. Pivkin, M.P. Kozochkin, A.N. Porvatov, J. Manuf. Mater. Process., 4(3), 96 (2020).

3. V. Grechishnikov, S. Grigoriev, P. Pivkin, M. Volosova, A. Isaev, D. Nikitin, I. Minin, EPJ Web Conf. 224, 05001 (2019).

4. B. Wang, Z. Liu, Int. J. Refract, Metals Hard Mater., 55, 24-32 (2016).

5. Fan Yinsheng, Bai Jicheng, Li Qiang, Li Chaojiang, Cao Yan, Li Zhengkai, Procedia CIRP, 42, 28 - 33 (2016).

6. M. Hogg, I. Timoshkin, S. MacGregor, M. Given, M. Wilson, T. Wang, IEEE, 2014, 391-394 (2014).

7. R.S. Khmyrov, S.N. Grigoriev, A.A. Okunkova, A.V. Gusarov, Phys. Procedia, 56, 345 (2014).

8. A.S. Metel, V.P. Bolbukov, M.A. Volosova, S.N. Grigoriev, Yu.A. Melnik, Instrum. Exp. Tech., 57(3), 345 (2014).

9. S.N. Grigoriev, G.M. Martinov, Procedia CIRP, 46, 525 (2016).

10. V.A. Grechishnikov, Y.E. Petukhov, P.M. Pivkin, et al., Meas Tech., 58, 848-853 (2015).

11. V.V. Kuzin, S.N. Grigoriev, M.A. Volosova, J. Frict. Wear, 35(6), 505 (2014).

12. S.N. Grigoriev, M.A. Volosova, A.A. Okunkova, S.V. Fedorov, K. Hamdy, P.A. Podrabinnik, P.M. Pivkin, M.P. Kozochkin, A.N. Porvatov, Technologies, 8(3), 49 (2020).

13. S.N. Grigoriev, D.A. Masterenko, V.I. Teleshevskii, P.N. Emelyanov, Meas. Tech., 55(11), 1311 (2013).

14. M.A. Volosova, S.N. Grigor'ev, V.V. Kuzin, Refract. Ind. Ceram., 56, 197, (2015).

15. A.S. Metel, V.P. Bolbukov, M.A. Volosova, S.N. Grigoriev, Yu.A. Melnik, Instrum. Exp. Tech., 57(3), 345 (2014).

16. M. Gostimirovic, P. Kovac, M. Sekulic, Sadhana Acad. Proc. Eng. Sci., 43(5), (2018).

17. S.N. Grigoriev, M.P. Kozochkin, A.N. Porvatov, P.S. Zhavoronsky, Jiang Xiaohui, P.M. Pivkin, EPJ Web Conf., 224, 05002 (2019).

18. V.V. Kuzin, S.N. Grigoriev, M.A. Volosova, J. Frict. Wear 36(1), 40 (2015).

19. D.S. Rechenko, A.G. Kol'tsov, Russian Engineering Research, 32(2), 179 - 181 (2012)

20. M. Volosova, S. Grigoriev, A.Metel, A. Shein, Coatings, 8, 287 (2018).

21. P.A. Nikishechkin, N.S. Grigoriev, N.Yu. Chervonnova. MATEC Web of Conf., 298, 00064 (2019).

22. X. Chen, Z. Wang, J. Xu, et al., J. Clean. Prod., 188, 1 - 11 (2018). 
23. S.N. Grigoriev, M.P. Kozochkin, P.M. Pivkin, IOP Conf. Ser.: Mater. Sci. Eng., 971, 022065 (2020)

24. S.N. Grigoriev, M.A. Volosova, A.A. Okunkova, S.V. Fedorov, K. Hamdy, P.A. Podrabinnik, A.N. Porvatov, Technologies, 8(3), 49 (2020).

25. S.N. Grigoriev, M.A. Volosova, A.A. Okunkova, S.V. Fedorov, K. Hamdy, P.A. Podrabinnik, A.N. Porvatov J. Manuf. Mater. Process., 4(3), 96 (2020).

26. S.N. Grigoriev, G.M. Martinov, Procedia CIRP, 1(1), 238 (2012).

27. I. A. Kovalev, P. A. Nikishechkin and A. S. Grigoriev. Approach to programmable controller building by its main modules synthesizing based on requirements specification for industrial automation. In: 2017 International Conference on Industrial Engineering, Applications and Manufacturing (ICIEAM), St. Petersburg, pp.1-4 (2017)

28. C. Zhang, R. Zou, Z. Yu, et al., Int J Adv Manuf Technol, 106, 5269-5276 (2020).

29. M.A. Volosova, S.N. Grigor'ev, V.V. Kuzin, Refract. Ind. Ceram., 55, 487 (2015).

30. YC. Lin, JC. Hung, HM. Lee, et al., Int J Adv Manuf Technol, 92, 2801-2808 (2017).

31. X. Liu et al., IEEE Transactions on Plasma Science, 43(6), 1959-1968 (2015).

32. M.S. Sohani, V.N. Gaitonde, B. Siddeswarappa, et al., Int J Adv Manuf Technol 45, 1131 (2009).

33. A. Mohd, S.G. Darius, Md. F. Bahari, Int. J. Mach. Tools Manuf., 47(7-8), 1214-1228 (2007).

34. X. Wang, Z. Liu, M. Qiu, Z. Hui, Z. Tian, Y. Huang, Mater. Manuf. Process., 29(11-12), 13671373 (2014). 